\title{
ESTIMATION OF THE ANTIOXIDANT ACTIVITY OF THE COMMERCIALLY AVAILABLE FERMENTED MILKS*
}

\author{
Dorota Najgebauer-Lejko ${ }^{\bowtie}$, Marek Sady \\ Department of Animal Product Technology, University of Agriculture in Krakow \\ Balicka 122, 30-149 Krakow, Poland
}

\begin{abstract}
Background. Free radicals are connected with the increased risk of certain diseases, especially cancers. There is some scientific evidence that antioxidant-rich diet may inhibit the negative impact of free radicals. The aim of the present study was to analyse the antioxidant capacity of the selected commercial natural and flavoured fermented milks offered in Poland, derived from different producers.

Material and methods. The following commercially available natural fermented milks: 12 yoghurts, 12 kefirs, 2 butter milks, 2 cultured milks, Turkish yoghurt drink (ayran) and the following flavoured fermented milks: 22 yoghurts, 2 acidophillus milks, 2 kefirs, butter milk and vegetable flavoured fermented milk were analysed for their antioxidant potential. The antioxidant capacity was assessed, in two replicates and twice for each product, by means of ferric reducing antioxidant power (FRAP) and DPPH radical scavenging ability (expressed as ARP - anti radical power) methods.

Results. Among all analysed plain products, yoghurts and kefirs were characterised by the highest antioxidant activity. The presence of probiotic Lactobacillus casei strains in the product positively affected both FRAP and ARP values. Antioxidant capacity of the flavoured fermented milks was primarily affected by the type and quality (e.g. fruit concentration) of the added flavouring preparation. The most valuable regarding the estimated parameters were chocolate, coffee, grapefruit with green tea extract as well as bilberry, forest fruits, strawberry and cherry with blackcurrant fillings.

Conclusions. Protein content, inclusion of probiotic microflora as well as type and quality of flavouring preparations are the main factors affecting antioxidant properties of fermented milks.
\end{abstract}

Key words: yoghurt, kefir, butter milk, probiotics, antioxidant activity

\section{INTRODUCTION}

Fermented milks, especially yoghurt, are very popular products consumed all over the word due to their excellent taste as well as nutritive and health-promoting properties. They are commercially available in different forms and types. This diversity may result from different basic chemical composition, i.e., fat and solids non-fat (SNF) content, method of production, the presence or absence of additional probiotic microbiota. They can be also produced as plain products or with addition of flavouring fillings (Grochulska, 2008; Tamime and Robinson, 2009). Besides the main goal of the fruit and non-fruit (chocolate, vanilla etc.) filling application, which is to impart the proper, desired sensory properties, fermented milks are also frequently

\footnotetext{
* Supported by the Ministry of Science and Higher Education, Poland, Project No. DS-3700/WTŻ.

『d.najgebauer-lejko@ur.krakow.pl, phone +48 126624805 
supplemented with bioactive ingredients, e.g. caffeine, guarana, green tea extract, Q10 coenzyme, ginseng, aloe vera, cranberry, dietary fiber, omega-3 fatty acids, phytosterols and phytostanols as well as prebiotic oligosaccharides (Cossu et al., 2009; Stankiewicz, 2009). Many of these ingredients show strong antioxidant capacity, which results mainly from the high content of polyphenolic compounds and antioxidant vitamins (C, E, carotenoids). There have been many studies performed which confirm that diet rich in antioxidants contributes to the improved protection of human organism against the negative action of free radicals, and thus to the decreased risk of many diseases, including certain types of cancer and cardiovascular disorders (Cossu et al., 2009; Jiménez et al., 2008; O'Rell and Chandan, 2006).

The most popular types of fermented milks in Poland are yoghurt, kefir and butter milk. According to the results of the examination of the consumers' preferences conducted in Krakow and Wrocław, the major factors which determined the selection of the fermented milks were: taste (approximately $25 \%$ of indications), brand (app. 24\%) and price (11-18\%), whereas less important were the parameters connected with the quality, i.e. nutritional value, fat content, health-promoting properties (Cymanow, 2008; Nowak et al., 2007). The highest share in the yoghurt market have the following manufacturers: Danone, Bakoma, Zott $(\sim 83 \%)$, whereas in the case of kefirs and butter milks also other brands like OSM Krasnystaw, Mlekpol and private brands play an important role (Cymanow, 2008; Grochulska, 2008; Nowak et al., 2007). Flavoured yoghurts are the most preferred by the consumers, and among this type the most popular are stawberry (above 50\% responses), followed by bilberry, peach, raspberry and other flavours (Cymanow, 2008). O'Rell and Chandan (2006) among ten the most prefered yoghurt flavours mentioned: strawberry, vanilla, pech, raspberry, strawberry-banana, natural, blueberry, lemon-lime, cherry, mixed berry. Contrary to yoghurts, the most preferable flavour of kefir and butter milk is the natural one (Grochulska, 2008).

The aim of the present work was to assess the antioxidant properties of certain natural and flavoured fermented milks available on the market in Poland.

\section{MATERIAL AND METHODS}

Fermented milks were bought in super- and hypermarkets in Krakow. The analyses were performer on the following natural products: 12 yoghurts, 12 kefirs, 2 butter milks, 2 cultured milks and Turkish yoghurt drink (ayran) and on the following flavoured fermented milks: 22 yoghurts, 2 acidophillus milks, 2 kefirs, 1 butter milk and vegetable flavoured fermented milk. Detailed list of the analysed natural and flavoured fermented milks as well as their composition and nutritional value (based on the information given by the producers on the label) are presented in Tables 1-3, respectively.

Fermented milks were screened for their antioxidant potential by means of two methods, i.e. ferric reducing antioxidant power (FRAP) and DPPH (2,2-diphenyl-1-picrylhydrazyl) radical scavenging ability methods. Both analyses were performed by the procedures described by Najgebauer-Lejko et al. (2011). The results of FRAP analysis were given as $\mathrm{mM} \mathrm{Fe}{ }^{2+} / \mathrm{dm}^{3}$ sample, whereas DPPH radical scavenging activity as ARP (anti radical power) expressed in relation to ARP of Trolox (6-hydroxy-2,5,7,8-tetramethylchroman-2-carboxylic acid) in mM TE (Trolox equivalents) per $1 \mathrm{~kg}$ sample. The antioxidant capacity was assessed, in two replicates and twice for each product (each product was bought twice in two different markets).

All results were subjected to the one-factor analysis of variance (ANOVA) and when applicable Duncan's test was performed to estimate the significance of difffences between mean values at $p \leq 0.05$ using Statistica 8.0 software (StatSoft, USA).

\section{RESULTS AND DISCUSSION}

Antioxidant properties of natural fermented milks result from the presence of antioxidant compounds in milk, i.e. casein, whey proteins, peptides and amino acids, CLA, uric acid, vitamin C, A and carotenoids, coenzyme $\mathrm{Q}_{10}$, enzymatic systems (superoxide dismutase, catalase, glutathione peroxidase) as well as from the antioxidant activity of the lactic acid bacteria or kefir culture (Jiménez et al., 2008; Liu et al., 2005; Pihlanto, 2006; Zulueta et al., 2009). Mean FRAP values $\left(\mathrm{mM} \mathrm{Fe}^{2+} / \mathrm{dm}^{3}\right)$ of the particular types of natural fermented milks amounted to respectively 
Najgebauer-Lejko, D., Sady, M. (2015). Estimation of the antioxidant activity of the commercially available fermented milks. Acta Sci. Pol. Technol. Aliment., 14(4), 387-396. DOI: 10.17306/J.AFS.2015.4.38

Table 1. Composition of natural yoghurts (YN), kefirs (KN), butter milks (BM), cultured milks (CM), Turkish drink "ayran" (by manufacturers)

\begin{tabular}{|c|c|c|}
\hline Product & Components other than milk and characteristic cultures & $\begin{array}{l}\text { Chemical composition } \\
\mathrm{g} / 100 \mathrm{~g}\end{array}$ \\
\hline YN1 & $\begin{array}{l}\text { DMP, MPr, dietary fiber from chicory } 3 \% \text {, Ca } 0.24 \% \text {; L. acidophilus LA-5, Bifidobacte- } \\
\text { rium } \mathrm{BB}-12\end{array}$ & $\mathrm{P}-4.5 ; \mathrm{C}-6.0 ; \mathrm{F}-2.0$ \\
\hline YN2 & DMP & $\mathrm{P}-3.4 ; \mathrm{C}-5.2 ; \mathrm{F}-2.7$ \\
\hline YN3 & MP, L. acidophilus, Bifidobacterium & $\mathrm{P}-3.6 ; \mathrm{C}-5.2 ; \mathrm{F}-3.0$ \\
\hline YN4 & $\mathrm{MP}, \mathrm{MPC}$ & $\mathrm{P}-5.1 ; \mathrm{C}-7.2 ; \mathrm{F}-2.0$ \\
\hline YN5 & DMP & $\mathrm{P}-4.6 ; \mathrm{C}-6.1 ; \mathrm{F}-2.0$ \\
\hline YN6 & DMP, MPr & $\mathrm{P}-4.7 ; \mathrm{C}-4.7 ; \mathrm{F}-3.0$ \\
\hline YN7 & MP, MPr, pectins, emulsifier E471 & $\mathrm{P}-4.4 ; \mathrm{C}-6.2 ; \mathrm{F}-3.5$ \\
\hline YN8 & PRDM, sugar or glucose syrup, glucose; L. casei Defensis (DN-114001) & $\mathrm{P}-2.8 ; \mathrm{C}-10.5 ; \mathrm{F}-1.6$ \\
\hline YN9 & $\begin{array}{l}\text { PRDM, water, glucose, pectin, aspartame, acesulfame K, aroma, L. casei Defensis } \\
\text { (DN-114001) }\end{array}$ & $\mathrm{P}-2.8 ; \mathrm{C}-3.3 ; \mathrm{F}-0.05$ \\
\hline YN10 & DMP, MPr, Bifidobacterium DN173010 & $\mathrm{P}-4.5 ; \mathrm{C}-5.1 ; \mathrm{F}-3.4$ \\
\hline YN11 & MP, L. acidophilus, Bifidobacterium, L. casei & $\mathrm{P}-4.3 ; \mathrm{C}-5.8 ; \mathrm{F}-1.5$ \\
\hline YN12 & $\mathrm{MP}, \mathrm{MPr}$ & $\mathrm{P}-5.0 ; \mathrm{C}-7.2 ; \mathrm{F}-3.0$ \\
\hline KN1 & L. acidophilus, Bifidobacterium & $\mathrm{P}-3.0 ; \mathrm{C}-4.0 ; \mathrm{F}-3.0$ \\
\hline KN2 & MP, L. acidophilus, Bifidobacterium & $P-3.6 ; C-5.5 ; F-1.8$ \\
\hline KN3 & OMP, MPr & $\mathrm{P}-3.2 ; \mathrm{C}-4.8 ; \mathrm{F}-1.4$ \\
\hline KN4 & MP & $\mathrm{P}-4.0 ; \mathrm{C}-4.7 ; \mathrm{F}-2.0$ \\
\hline KN5 & DMP & $P-3.6 ; C-5.5 ; F-2.0$ \\
\hline KN6 & nd & $* \mathrm{P}-2.93 \% ; \mathrm{F}-1.5 \%$ \\
\hline KN7 & MP, MPr & $\mathrm{P}-4.0 ; \mathrm{C}-5.7 ; \mathrm{F}-2.0$ \\
\hline KN8 & nd & $\mathrm{P}-3.2 ; \mathrm{C}-4.0 ; \mathrm{F}-1.5$ \\
\hline KN9 & DMP, MPr & $\mathrm{P}-4.0 ; \mathrm{C}-4.8 ; \mathrm{F}-0.0$ \\
\hline KN10 & MPr & $\mathrm{P}-3.9 ; \mathrm{C}-5.7 ; \mathrm{F}-1.5$ \\
\hline KN11 & MPr, DMP & $\mathrm{P}-3.9 ; \mathrm{C}-5.0 ; \mathrm{F}-0.05$ \\
\hline KN12 & nd & $\mathrm{P}-3.4 ; \mathrm{C}-4.6 ; \mathrm{F}-2.0$ \\
\hline BM 1 & DMP, MPr & $\mathrm{P}-3.5 ; \mathrm{C}-4.5 ; \mathrm{F}-2.0$ \\
\hline BM 2 & DMP, butter milk & $F-1.5$ \\
\hline CM1 & nd & $\mathrm{P}-3.3 ; \mathrm{C}-4.8 ; \mathrm{F}-2.0$ \\
\hline CM2 & nd & $\mathrm{P}-3.1 ; \mathrm{C}-4.4 ; \mathrm{F}-1.5$ \\
\hline Ayran & MPr, pectins, water; emulsifier E471, salt, lactic acid & $\mathrm{P}-2.7 ; \mathrm{C}-4.0 ; \mathrm{F}-2.2$ \\
\hline
\end{tabular}

Explanatory notes: MP - milk powder, DMP - defatted milk powder, MPr - milk proteins, PRDM - pasteurised reconstituted defatted milk, MPC - milk protein concentrate, $\mathrm{nd}$ - not declared, $\mathrm{P}$ - protein, $\mathrm{C}$ - carbohydrates, $\mathrm{F}$ - fat.

*Based on authors' own research. 
Najgebauer-Lejko, D., Sady, M. (2015). Estimation of the antioxidant activity of the commercially available fermented milks. Acta Sci. Pol. Technol. Aliment., 14(4), 387-396. DOI: 10.17306/J.AFS.2015.4.38

Table 2. Composition of the flavoured yoghurts (by manufacturers)

\begin{tabular}{|c|c|c|}
\hline Flavour & Active ingredients including additional microbiota & $\begin{array}{l}\text { Chemical composition } \\
\mathrm{g} / 100 \mathrm{~g}\end{array}$ \\
\hline Coffee & Instant coffee $0.3 \%$, cocoa, $L$. acidophilus, Bifidobacterium & $\mathrm{P}-2.3 ; \mathrm{C}-15.0 ; \mathrm{F}-8.0$ \\
\hline Coffee with guarana & Natural coffee extract $0.2 \% *$, guarana extract $0.04 \% *$, LA- 5, BB- 12 & $\mathrm{P}-2.8 ; \mathrm{C}-13.0 ; \mathrm{F}-0.8$ \\
\hline Aloe vera with omega 3 & Aloe vera - omega $3-3.3 \%$ ( $\alpha$-linolenic acid $-0.02 \%)$, LA-5, BB-12 & $\mathrm{P}-2.8 ; \mathrm{C}-13.0 ; \mathrm{F}-0.8$ \\
\hline Chocolate & Chocolate $3 \% *$, cocoa, cocoa butter, vitamins $\mathrm{A}_{\text {and }} \mathrm{D}_{3}$ & $\mathrm{P}-2.8 ; \mathrm{C}-13.0 ; \mathrm{F}-2.5$ \\
\hline Pink grapefruit with green tea & Pink grapefruit juice $1.2 \% *$, green tea extract $0.06 \% *$, LA-5, BB-12 & $\mathrm{P}-2.8 ; \mathrm{C}-13.0 ; \mathrm{F}-0.8$ \\
\hline Grapefruit with green tea & $\begin{array}{l}\text { Pink grapefruit - green tea filling } 4 \% \text { (grapefruit juice from concen- } \\
\text { trate } 25 \% * \text { ) }\end{array}$ & $\mathrm{P}-\mathrm{nd} ; \mathrm{C}-\mathrm{nd} ; \mathrm{F}-1.5$ \\
\hline Cherry-ginseng & Cherries $3 \%^{*}$, ginseng extract $0.02 \%^{*}$, LA-5, BB-12 & $\mathrm{P}-2.8 ; \mathrm{C}-13.0 ; \mathrm{F}-0.8$ \\
\hline Strawberry-apple-wild rose & $\begin{array}{l}\text { Fruit filling } 15 \% \text { (apple } 22 \% * \text {, strawberry } 20 \% \text {, wild rose hip juice } \\
\text { from concentrate } 15 \% * \text { ), Lb. acidophilus, Lb. paracasei, } \\
\text { Bifidobacterium lactis }\end{array}$ & $\mathrm{P}-\mathrm{nd} ; \mathrm{C}-\mathrm{nd} ; \mathrm{F}-1.5$ \\
\hline Forest fruits & $\begin{array}{l}\text { Raspberries } 2.7 \%^{*} \text {, blackberries } 1.4 \% * \text {, blueberry juice } 0.6 \% * \text {, } \\
\text { carrot and elderberry concentrate, Bifidobacterium DN173010 }\end{array}$ & $\mathrm{P}-2.8 ; \mathrm{C}-12.5 ; \mathrm{F}-1.6$ \\
\hline Bilberry 1 & Bilberries and bilberry juice from concentrate $6.3 \%$ & $\mathrm{P}-3.5 ; \mathrm{C}-15.0 ; \mathrm{F}-2.5$ \\
\hline Bilberry 2 & Bilberries $2 \%$, red beetroot juice concentrate, anthocyanins & $\mathrm{P}-2.9 ; \mathrm{C}-17.3 ; \mathrm{F}-1.1$ \\
\hline Dried plum 1 & $\begin{array}{l}\text { Dried plums } 3.2 \% * \text {, fresh plums }-0.2 \% * \text {, Bifidobacterium } \\
\text { DN173010 }\end{array}$ & $\mathrm{P}-2.8 ; \mathrm{C}-13.1 ; \mathrm{F}-1.6$ \\
\hline Dried plum 2 & Oligofructose, dried plum $-2.6 \%$, carrot concentrate & $\mathrm{P}-3.7 ; \mathrm{C}-14.9 ; \mathrm{F}-2.4$ \\
\hline Dried apricot & Oligofructose, dried apricots $1.9 \%$, carrot concentrate, beta-carotene & $\mathrm{P}-3.7 ; \mathrm{C}-15.4 ; \mathrm{F}-2.4$ \\
\hline Cherry-blackcurrant & $\begin{array}{l}\text { Cherries } 4.8 \% * \text {, blackcurrants } 0.5 \% * \text {, blackcurrant juice } 0.3 \% * \text {, } \\
\text { black carrot juice, Bifidobacterium DN173010 }\end{array}$ & $\mathrm{P}-2.8 ; \mathrm{C}-12.9 ; \mathrm{F}-1.6$ \\
\hline Cranberry & Cranberry $4 \% \%^{*}$, carrot concentrate, L. casei, vitamin C & $\mathrm{P}-2.9 ; \mathrm{C}-13.4 ; \mathrm{F}-2.0$ \\
\hline Peach & Peaches, peach and passion fruit juice from concentrate & $\mathrm{P}-3.5 ; \mathrm{C}-14.6 ; \mathrm{F}-2.5$ \\
\hline Strawberry 1 & $\begin{array}{l}\text { Strawberries and strawberry juice from concentrate } 9 \% \text {, } \\
\text { red beetroot juice concentrate }\end{array}$ & $\mathrm{P}-3.5 ; \mathrm{C}-15.0 ; \mathrm{F}-2.5$ \\
\hline Strawberry 2 & Strawberries $2 \%$ & $\mathrm{P}-2.9 ; \mathrm{C}-17.3 ; \mathrm{F}-1.1$ \\
\hline Strawberry 3 & Vitamins: folic acid, B6, B12 & $\mathrm{P}-1.8 ; \mathrm{C}-15.7 ; \mathrm{F}-1.0$ \\
\hline Apple-kiwi & $\begin{array}{l}\text { Fruit filling } 23 \% \text { (apple } 20 \% * \text {, kiwi } 14 \% \%^{*} \text {, apple juice } \\
\text { from concentrate } 8 \%^{*} \text { ) }\end{array}$ & $\mathrm{P}-3.0 ; \mathrm{C}-13.8 ; \mathrm{F}-3.6$ \\
\hline Pineapple & Pineapple and pineapple juice from concentrate $3 \%$ & $\mathrm{P}-3.5 ; \mathrm{C}-15.0 ; \mathrm{F}-2.5$ \\
\hline
\end{tabular}

Explanatory notes: $\mathrm{P}$ - protein, $\mathrm{C}$ - carbohydrates, $\mathrm{F}$ - fat, *content in fruit/chocolate preparation, $\mathrm{nd}$ - not declared.

(in decreasing order): 1.19 (yoghurts), 1.03 (kefirs), 0.66 (cultured milks), 0.48 (ayran) and 0.45 (butter milks). The highest ability to scavenge DPPH radicals were also found for yoghurts $(0.19 \mathrm{mM} \mathrm{TE} / \mathrm{kg})$ and kefirs $(0.17 \mathrm{mM} \mathrm{TE} / \mathrm{kg})$, followed by butter milks, cultured milks and ayran, for which the following mean ARP values were determined: 0.14, 0.11 and $0.09 \mathrm{mM}$ TE/kg (respectively; Table 4). These results indicate 
Najgebauer-Lejko, D., Sady, M. (2015). Estimation of the antioxidant activity of the commercially available fermented milks. Acta Sci. Pol. Technol. Aliment., 14(4), 387-396. DOI: 10.17306/J.AFS.2015.4.38

Table 3. Composition of the flavoured fermented milks (by manufacturers)

\begin{tabular}{|c|c|c|}
\hline Product & Ingredients (other than milk) & $\begin{array}{l}\text { Chemical composition } \\
\mathrm{g} / 100 \mathrm{~g}\end{array}$ \\
\hline Acidophilus milk-vanilla & $\begin{array}{l}\text { Vanilla-flavoured syrup } 15 \% \text { (sugar, water, aroma, colouring agents: } \\
\text { E160 C, E110), lactic acid bacteria, } L b \text {. acidophilus }\end{array}$ & $\mathrm{P}-3.0 ; \mathrm{C}-14.0 ; \mathrm{F}-2.0$ \\
\hline Acidophilus milk-cherry & $\begin{array}{l}\text { Cherry-flavoured syrup 15\% (sugar, water, cherry concentrate } 2 \% \text {, aroma, } \\
\text { colouring agents: E160 C, E120), lactic acid bacteria, Lb. acidophilus }\end{array}$ & $\mathrm{P}-3.0 ; \mathrm{C}-14.0 ; \mathrm{F}-2.0$ \\
\hline $\begin{array}{l}\text { Butter milk with straw- } \\
\text { berry and rhubarb }\end{array}$ & $\begin{array}{l}\text { Fruit filling } 8 \% \text { (fruits, sugar, glucose-fructose syrup, modified starch, } \\
\text { thickening agent: pectin, aroma, colouring agent: cochineal), defatted } \\
\text { milk powder, live bacteria culture }\end{array}$ & $\mathrm{P}-2.8 ; \mathrm{C}-9.6 ; \mathrm{F}-1.3$ \\
\hline Kefir with cranberry & $\begin{array}{l}\text { Cranberry syrup } 11 \% \text { (sugar, cranberry syrup } 2 \% \text {, carrot, grape } \\
\text { and chockeberry concentrate, aroma, cochineal), milk proteins, } \\
\text { live bacteria culture }\end{array}$ & $\mathrm{P}-3.3 ; \mathrm{C}-9.0 ; \mathrm{F}-0.05$ \\
\hline Strawberry kefir & $\begin{array}{l}\text { Fruit filling (sugar, strawberry puree, modified starch, citric acid, } \\
\text { cochineal, aromas) sugar, live lactic acid bacteria }\end{array}$ & $\mathrm{P}-2.8 ; \mathrm{C}-11.0 ; \mathrm{F}-1.5$ \\
\hline $\begin{array}{l}\text { Vegetable flavoured } \\
\text { fermented milk }\end{array}$ & $\begin{array}{l}\text { Vegetable filling: cucumber, red beetroot, garlic, dill, wheat glucose-fruc- } \\
\text { tose syrup, salt, modified maize starch, red beetroot concentrate, chive, } \\
\text { pepper, wine vinegar, aroma, sodium glutamate }\end{array}$ & $\mathrm{P}-3.2 ; \mathrm{C}-5.0 ; \mathrm{F}-1.8$ \\
\hline
\end{tabular}

Explanatory notes: $\mathrm{P}$ - protein, $\mathrm{C}$ - carbohydrates, $\mathrm{F}$ - fat.

Table 4. Antioxidant activity of the plain fermented milks (means \pm standard deviation, $n=4$ )

\begin{tabular}{lcc}
\hline Product & FRAP, $\mathrm{mM} \mathrm{Fe}^{2+} / \mathrm{dm}^{3}$ & ARP, $\mathrm{mM} \mathrm{TE} / \mathrm{kg}$ \\
\hline \multicolumn{1}{c}{2} & 3 \\
\hline Plain yoghurts & \\
\hline YN1 & $0.993^{\mathrm{bc}} \pm 0.050$ & $0.198^{\mathrm{a}} \pm 0.025$ \\
YN2 & $1.006^{\mathrm{bc}} \pm 0.512$ & $0.193^{\mathrm{a}} \pm 0.030$ \\
YN3 & $0.466^{\mathrm{c}} \pm 0.241$ & $0.123^{\mathrm{a}} \pm 0.014$ \\
YN4 & $2.445^{\mathrm{a}} \pm 0.028$ & $0.201^{\mathrm{a}} \pm 0.026$ \\
YN5 & $1.147^{\mathrm{bc}} \pm 0.231$ & $0.185^{\mathrm{a}} \pm 0.018$ \\
YN6 & $0.834^{\mathrm{bc}} \pm 0.194$ & $0.141^{\mathrm{a}} \pm 0.010$ \\
YN7 & $0.953^{\mathrm{bc}} \pm 0.303$ & $0.220^{\mathrm{a}} \pm 0.084$ \\
YN8 & $1.676^{\mathrm{ab}} \pm 0.587$ & $0.237^{\mathrm{a}} \pm 0.065$ \\
YN9 & $1.518^{\mathrm{b}} \pm 0.406$ & $0.240^{\mathrm{a}} \pm 0.013$ \\
YN10 & $0.776^{\mathrm{bc}} \pm 0.069$ & $0.176^{\mathrm{a}} \pm 0.051$ \\
YN11 & $1.358^{\mathrm{bc}} \pm 0.068$ & $0.166^{\mathrm{a}} \pm 0.009$ \\
YN12 & $1.071^{\mathrm{bc}} \pm 0.798$ & $0.170^{\mathrm{a}} \pm 0.028$ \\
\hline Plain kefirs & & \\
\hline KN1 & $1.228^{\mathrm{b}} \pm 0.312$ & $0.149^{\mathrm{a}} \pm 0.006$ \\
KN2 & $1.083^{\mathrm{bc}} \pm 0.175$ & $0.162^{\mathrm{a}} \pm 0.043$ \\
KN3 & $0.830^{\mathrm{bc}} \pm 0.157$ & $0.196^{\mathrm{a}} \pm 0.067$ \\
\hline
\end{tabular}

Table 4 - cont.

\begin{tabular}{lcc}
\hline \multicolumn{1}{c}{1} & 2 & 3 \\
\hline KN4 & $0.580^{\mathrm{c}} \pm 0.002$ & $0.242^{\mathrm{a}} \pm 0.128$ \\
KN5 & $0.992^{\mathrm{bc}} \pm 0.000$ & $0.161^{\mathrm{a}} \pm 0.030$ \\
KN6 & $0.653^{\mathrm{c}} \pm 0.133$ & $0.164^{\mathrm{a}} \pm 0.030$ \\
KN7 & $2.520^{\mathrm{a}} \pm 0.475$ & $0.135^{\mathrm{a}} \pm 0.038$ \\
KN8 & $0.819^{\mathrm{bc}} \pm 0.228$ & $0.149^{\mathrm{a}} \pm 0.011$ \\
KN9 & $0.965^{\mathrm{bc}} \pm 0.138$ & $0.207^{\mathrm{a}} \pm 0.014$ \\
KN10 & $0.695^{\mathrm{bc}} \pm 0.045$ & $0.151^{\mathrm{a}} \pm 0.006$ \\
KN11 & $1.237^{\mathrm{b}} \pm 0.357$ & $0.177^{\mathrm{a}} \pm 0.003$ \\
KN12 & $0.760^{\mathrm{bc}} \pm 0.193$ & $0.123^{\mathrm{a}} \pm 0.009$ \\
\hline Butter milks $(\mathrm{BM})$, cultured milks $(\mathrm{CM}),^{\mathrm{a}} \mathrm{ayran}$ \\
\hline BM1 & $0.235^{\mathrm{a}} \pm 0.150$ & $0.131^{\mathrm{a}} \pm 0.027$ \\
BM2 & $0.628^{\mathrm{a}} \pm 0.151$ & $0.140^{\mathrm{a}} \pm 0.036$ \\
CM1 & $0.635^{\mathrm{a}} \pm 0.088$ & $0.068^{\mathrm{a}} \pm 0.001$ \\
CM2 & $0.688^{\mathrm{a}} \pm 0.692$ & $0.156^{\mathrm{a}} \pm 0.005$ \\
Ayran & $0.482^{\mathrm{a}} \pm 0.003$ & $0.092^{\mathrm{a}} \pm 0.063$ \\
\hline
\end{tabular}

Explanatory notes: FRAP - ferric reducing antioxidant power, ARP - antiradical power (against DPPH radical), different superscript letters in columns and in the certain product group denote statistically significant differences at $p \leq 0.05$. 
that among all types of fermented milks yoghurts and kefirs were characterised by the highest antioxidant capacity. This phenomenon may be connected with the fact that these types of fermented milk products are very often fortified with milk powder and/or milk proteins. Manufacturers declared addition of milk protein preparations into all analysed plain yoghurt and 8 of 12 kefirs (Table 1). According to many reports milk proteins, especially rich in sulphur and hydrophobic amino acids whey proteins, are potent antioxidant substances (Cichosz et al., 2012; Pihlanto, 2006). Another factor that should be taken into account is the supplementation of the fermented milks, particularly yoghurts with additional probiotic bacteria. Data found in literature indicate that certain Lactobacillus and Bifidobacterium strains exhibit significant antioxidant properties (Zhang et al., 2010). In the present work, among all studied plain yoghurts the highest FRAP values (1.4-2.4 $\left.\mathrm{mM} \mathrm{Fe}^{2+} / \mathrm{dm}^{3}\right)$ were observed (in decreasing order) for: YN4, YN8, YN9 and YN11 (Table 4). The first mentioned yoghurt according to data provided by the producer was characterised by the highest protein content $(5.1 \%$, fortified with milk protein concentrate), whereas the following three yoghurts contained $L$. casei bacteria. Analysing the results for ARP values in the group of the natural fermented milks one can conclude that there was lower diversity of the results (differences not statistically significant, $p>0.05$ ) when compared to FRAP values. The radical scavenging ability in this group was estimated in the range of $0.07-0.24 \mathrm{mM} \mathrm{TE} / \mathrm{kg}$, and the highest values were noticed for YN8 and YN9 yoghurts (both containing probiotic $L$. casei $\mathrm{DN}-114001$ strain) and for the KN4 kefir. Antioxidant activity of $L$. casei bacteria was confirmed in both in vitro (Virtanen et al., 2007) and in vivo studies (Kapila and Sinha, 2006; Zhang et al., 2010). Moreover, the results obtained by Jain et al. (2009) revealed that among three tested lactic acid bacteria (LAB) strains, the culture of $L$. casei was characterised by the highest ability to eliminate DPPH, hydrogen peroxide, malonaldialdehyde radicals, and to inhibit linoleic acid peroxidation. Many studies indicate that the antioxidant potential of LAB is influenced not only by the species but also by the strain of the bacteria, therefore careful choice of the culture for milk inoculation may be helpful in designing products with additional health-promoting properties (Saide and Gilliand, 2005; Virtanen et al., 2007).

The diversification of the results obtained for flavoured fermented milks was much higher than for the natural products. The obtained FRAP and ARP values in the former group were also higher when compared to the latter products (Table 5). The antioxidant properties of the flavoured yoghurts were on average two-fold (FRAP) and five-fold (ARP) higher than the respective values stated for the yoghurts without flavouring fillings. This resulted from the fact that fruits, tea, coffee, chocolate (cocoa) are known for their distinguishing high antioxidant potential (Halvorsen et al., 2006). This is also consistent with the results obtained by Carlsen et al. (2010), who estimated the FRAP values of the natural, strawberry and mixed wild berries yoghurts at the level of respectively: 0.05 ; $0.11 ; 0.25 \mathrm{mM} / 100 \mathrm{~g}$.

Antioxidant properties of the flavoured fermented milks were in the greatest manner influenced by the type of applied filling and its quality. FRAP values in the group of flavoured yoghurts fluctuated from 0.72 (peach yoghurt) to $7.48 \mathrm{mM} \mathrm{Fe}{ }^{2+} / \mathrm{dm}^{3}$ (coffee yoghurt), whereas for other fermented milks (acidophilus milks, kefirs, butter milk, vegetable flavoured fermented milk) they were in the range of $0.66-1.64 \mathrm{mM}$ $\mathrm{Fe}^{2+} / \mathrm{dm}^{3}$. The strongest ability to reduce $\mathrm{Fe}^{3+}$ to $\mathrm{Fe}^{2+}$ ions and to scavenge DPPH radicals was determined for the yoghurts with coffee extracts and chocolate, whereas less potent, however still exhibiting high antioxidant capacity, were those supplemented with green tea extracts. Among fruit yoghurts the following supplements affected the highest antioxidant potential: bilberry (1), forest fruits, strawberry (1) and cherryblackcurrant. On the contrary, yoghurts containing light-coloured fruits (peach, apricot, pineapple) were characterised with lower FRAP and ARP values. These results are in agreement with the findings of Halvorsen et al. (2006), who listed chocolate, strawberries, bilberries and coffee among the 50 food products with the highest antioxidant capacity. So strong antioxidant properties of fruits result mainly from the considerable content of polyphenols, vitamin $\mathrm{C}$ and to a lesser extent from the presence of vitamins A, B and carotenoids (Lim et al., 2007). Skrede et al. (2004), while studying different fruit preparations destined for application in dairy products observed the highest ability 
Table 5. Antioxidant activity of flavoured fermented milks (means \pm standard deviation, $n=4$ )

\begin{tabular}{|c|c|c|}
\hline Product (flavour) & FRAP, $\mathrm{mM} \mathrm{Fe}^{2+} / \mathrm{dm}^{3}$ & ARP, mM TE/kg \\
\hline \multicolumn{3}{|l|}{ Flavoured yoghurts } \\
\hline Coffee & $7.48^{i} \pm 0.01$ & $2.20^{\mathrm{hi}} \pm 0.22$ \\
\hline Coffee with guarana & $3.96^{\mathrm{g}} \pm 0.32$ & $1.49^{\mathrm{fgh}} \pm 0.30$ \\
\hline Chocolate & $5.73^{\mathrm{h}} \pm 0.10$ & $2.43^{\mathrm{i}} \pm 0.09$ \\
\hline Aloe vera with omega 3 & $0.90^{\mathrm{ab}} \pm 0.03$ & $0.66^{\mathrm{abc}} \pm 0.01$ \\
\hline Pink grapefruit with green tea & $2.4^{\text {cdef }} \pm 1.25$ & $1.26^{\text {efgh }} \pm 0.39$ \\
\hline Grapefruit with tea & $3.30^{\mathrm{fg}} \pm 0.28$ & $0.22^{\mathrm{a}} \pm 0.02$ \\
\hline Cherry-ginseng & $1.67^{\mathrm{abcd}} \pm 0.70$ & $0.50^{\mathrm{abc}} \pm 0.04$ \\
\hline Strawberry-apple-wild rose & $1.30^{\mathrm{abc}} \pm 0.07$ & $0.72^{\mathrm{abcd}} \pm 0.06$ \\
\hline Forest fruits & $2.83^{\mathrm{ef}} \pm 0.50$ & $1.54^{\mathrm{fg}} \pm 0.50$ \\
\hline Bilberry 1 & $3.35^{\mathrm{gh}} \pm 1.12$ & $1.71^{\mathrm{gh}} \pm 0.15$ \\
\hline Bilberry 2 & $1.20^{\mathrm{ab}} \pm 0.02$ & $0.62^{\mathrm{abc}} \pm 0.10$ \\
\hline Dried plum 1 & $1.96^{\text {bcde }} \pm 0.57$ & $0.77^{\mathrm{abcd}} \pm 0.59$ \\
\hline Dried plum 2 & $1.65^{\mathrm{abcd}} \pm 0.02$ & $0.67^{\mathrm{abc}} \pm 0.01$ \\
\hline Dried apricot & $1.45^{\mathrm{abcd}} \pm 0.01$ & $0.47^{\mathrm{ab}} \pm 0.01$ \\
\hline Cherry-blackcurrant & $3.08^{\mathrm{gh}} \pm 0.07$ & $0.94^{\text {bcde }} \pm 0.50$ \\
\hline Cranberry & $1.31^{\mathrm{abc}} \pm 0.08$ & $0.78^{\mathrm{abcd}} \pm 0.17$ \\
\hline Peach & $0.72^{\mathrm{a}} \pm 0.04$ & $0.47^{\mathrm{ab}} \pm 0.09$ \\
\hline Strawberry 1 & $2.45^{\mathrm{efg}} \pm 0.61$ & $1.08^{\mathrm{cdef}} \pm 0.01$ \\
\hline Strawberry 2 & $1.16^{\mathrm{ab}} \pm 0.28$ & $0.74^{\mathrm{abcd}} \pm 0.34$ \\
\hline Strawberry 3 & $1.30^{\mathrm{abc}} \pm 0.01$ & $0.40^{\mathrm{ab}} \pm 0.07$ \\
\hline Apple-kiwi & $1.32^{\mathrm{ab}} \pm 0.09$ & $0.66^{\mathrm{abc}} \pm 0.06$ \\
\hline Pineapple & $1.49^{\mathrm{abcd}} \pm 0.49$ & $0.55^{\mathrm{abc}} \pm 0.11$ \\
\hline
\end{tabular}

Flavoured acidophilus milks (AM), kefirs (K), butter milks (M), vegetable flavoured fermented milk

\begin{tabular}{lrc}
\hline Vanilla - AM & $0.99^{\mathrm{a}} \pm 0.02$ & $0.12^{\mathrm{a}} \pm 0.09$ \\
Cherry - AM & $0.66^{\mathrm{a}} \pm 0.03$ & $0.24^{\mathrm{a}} \pm 0.05$ \\
Strawberry - K & $1.16^{\mathrm{ac}} \pm 0.06$ & $0.58^{\mathrm{a}} \pm 0.46$ \\
Cranberry - K & $1.59^{\mathrm{b}} \pm 0.10$ & $0.34^{\mathrm{a}} \pm 0.15$ \\
Strawberry with rhubarb - M & $1.64^{\mathrm{b}} \pm 0.04$ & $0.88^{\mathrm{a}} \pm 0.03$ \\
Vegetable flavoured fermented milk & $1.41^{\mathrm{bc}} \pm 0.27$ & $0.20^{\mathrm{a}} \pm 0.13$ \\
\hline
\end{tabular}

Explanatory notes as under the Table 4. 
to scavenge DPPH radicals (ARP) and oxygen radical absorbance capacity (ORAC) for the fillings prepared from berry fruits. High content of polyphenolic compounds, especially red/bluish-coloured anthocyanins is considered to be a key factor contribuiting to the antioxidant activity of berries. The authors observed also 5-13-fold increase of the ARP values for the probiotic milks blended with $13 \%$ of bilberry and blackcurrant extracts when compared to the plain fermented milk sample.

The results of the present study indicate that among different yoghurts of the same flavours (strawberry, bilberry) the products containing lower share of fruits (cheaper ones) exhibited 50\% (or less) of the antioxidant activity measured for the yoghurts with richer composition. This relation is particularly evident for strawberry yoghurts, which showed the ARP values in the following order: Strawberry $1>$ Strawberry $2>$ Strawberry 3 , and the obtained FRAP values decreased in the order: Strawberry $1>$ Strawberry 3 and Strawberry 2 . The fruit content in these products, according to the manufacturers amounted to: $9 \%$ (Strawberry 1), $2 \%$ (Strawberry 2), or the product did not contain fruits (Strawberry 3, flavour and colour imparted by an addition of aromas and carmine). The differences in the antioxidant parameters were observed also for the two tested bilberry yoghurts. These fermented milks (both derived from the same producer) contained respectively 6.3 and $2 \%$ of fruits, what resulted in almost 3 -fold higher DPPH radical scavenging ability and ferric reducing power of the former product. According to the study of Carlsen et al. (2010) three tested strawberry yoghurts differed as regards measured FRAP values which ranged from 0.08 to $0.13 \mathrm{mM} / 100 \mathrm{~g}$, and the highest value was determined for the yoghurt with fruits at the bottom of a cup. In the group of flavoured fermented milks other than yoghurts, the lowest antioxidant parameters were observed for acidophilus milks. In this case the differences could result from the quality of the flavouring preparation. Commercial flavouring fillings for fermented milks are offered in the variety of types, e.g. fillings with the higher share of fruits, prepared on the basis of fruit pulps or purees, or cheaper fillings in the form of syrups usually apllied for drinkable fermented milks (Şengül et al., 2012; Zaręba and Ziarno, 2012). The proper taste and odour of the fermented milk can be also achieved by the application of the synthetic flavouring substances (Coïsson et al., 2005). Both studied acidophilus milks were flavoured with respectively vanilla and cherry syrups, what was reflected in the level of measured parameters. On the other hand, strawberry kefir and butter milk were characterised with the ARP and FRAP values similar to those determined for the strawberry yoghurts. The only product enriched with vegetables and herbs showed FRAP value close to the average level of this parameter obtained for fruit kefirs, butter milk and most of the fruit yoghurts.

\section{CONCLUSIONS}

Among natural fermented milks, the most important factors positively inluencing antioxidant capacity were high protein content and the presence of additional probiotic microflora of the $L$. case $i$ species. On the other hand, in the group of flavoured fermented milks the addition of chocolate, coffee, green tea extract, and dark-coloured fruits (bilberries, forest fruits, blackcurrants, cherries, strawberries) imparted strong antioxidant properties. The quality of the flavouring filling, i.e., the content of bioactive components, was also of a great importance. Summarizing, the results of the present study indicate that measuring of the antioxidant capacity can be considered as an another tool which allows to control the quality of fermented milks.

\section{REFERENCES}

Carlsen, M. H., Halvorsen, B. L., Holte, K., Bohn, S. K., Dragland, S., Sampson, L., ..., Willey, C. (2010). The total antioxidant content of more than 3100 foods, beverages, spices, herbs and supplements used worldwide. Nutr. J., 9(3), 1-11.

Cichosz, G., Ambroziak, A., Kowalska, M., Aljewicz, M. (2012). Składniki mleka o działaniu antynowotworowym [Milk ingredients exhibiting anticancer properties]. Przegl. Mlecz., 3, 4-10 [in Polish].

Coïsson, J. D., Travaglia, F., Piana, G., Capasso, M., Arlorio, M. (2005). Euterpe oleracea juice as a functional pigment for yogurt. Food Res. Int., 38(8), 893-897.

Cossu, M., Juliano, C., Pisu, R., Alamanni, M. C. (2009). Effects of enrichment with polyphenolic extracts from Sardinian plants on physico-chemical, antioxidant and microbiological properties of yogurt. Ital. J. Food Sci., 4(21), 447-459. 
Cymanow P. (2008). Ocena preferencji konsumentów produktów mleczarskich na przykładzie mieszkańców Krakowa [Evaluation of consumer preferences for dairy products on the ex ample of Cracow's residents]. Rocz. Nauk. SERiA, 10(4), 51-55 [in Polish].

Grochulska, C. (2008). Mleczna droga - raport o produktach mlecznych [Marketing milk - report on dairy products]. Fresh Cool Market, 05(08), 18-25 [in Polish].

Halvorsen, B. L., Carlsen, M. H., Phillips, K. M., Bøhn, S. K., Holte, K., Jacobs, D. R., Blomhoff, R. (2006). Content of redox-active compounds (ie, antioxidants) in foods consumed in the United States. Am. J. Clin. Nutr., 84(1), 95-135.

Jain, S., Yadav, H., Ravindra, Sinha, P. (2009). Antioxidant and cholesterol assimilation activities of selected lactobacilli and lactococci cultures. J. Dairy Res., 76(4), 385-391.

Jiménez, A. M., Murcia, M. A., Parras, P., Martínez-Tomé, M. (2008). On the importance of adequately choosing the ingredients of yoghurt and enriched milk for their antioxidant activity. Int. J. Food Sci. Tech., 43(8), 1464-1473.

Kapila, S., Sinha, P. R. (2006). Antioxidative and hypocholesterolemic effect of Lactobacillus casei ssp casei (biodefensive properties of lactobacilli). Indian J. Med. Sci., 60(9), 361.

Lim, Y. Y., Lim, T. T., Tee, J. J. (2007). Antioxidant properties of several tropical fruits: A comparative study. Food Chem., 103(3), 1003-1008.

Liu, J. R., Lin, Y. Y., Chen, M. J., Chen, L. J., Lin, C. W. (2005). Antioxidative activities of kefir. Asian-Aust. J. Anim. Sci., 18(4), 567-573.

Najgebauer-Lejko, D., Sady, M., Grega, T., Walczycka, M. (2011). The impact of tea supplementation on microflora, $\mathrm{pH}$ and antioxidant capacity of yoghurt. Int. Dairy J., 21(8), 568-574.

Nowak, M., Trziszka, T., Szołtysik, M. (2007). Preferencje konsumentów mlecznych napojów fermentowanych [Consumers' preferences of fermented milk drinks]. Żywn. Nauka Techn. Jakość, 1(50), 77-83 [in Polish].

O’Rell, K. R., Chandan, R. C. (2006). Yogurt: Fruit preparations and flavoring materials. In R. C. Chandan, C. H. White, A. Kilara, Y. H. Hui (Eds.), Manufacturing yogurt and fermented milks (pp. 151-166). USA: Blackwell Publishing.

Pihlanto, A. (2006). Antioxidative peptides derived from milk proteins. Int. Dairy J., 16, 1306-1314.

Saide, J. A. O., Gilliand, S. E. (2005). Antioxidative activity of lactobacilli measured by oxygen radical absorbance capacity. J. Dairy Sci., 88(4), 1352-1357.

Şengül, M., Erkaya, T., Şengül, M., Yildiz, H. (2012). The effect of adding sour cherry pulp into yoghurt on the physicochemical properties, phenolic content and antioxidant activity during storage. Int. J. Dairy Technol., 65(3), 429-436.

Skrede, G., Bryhn, Larsen, V., Aaby, K., Jørgensen, A. S., Birkeland, S. E. (2004). Antioxidative properties of commercial fruit preparations and stability of bilberry and black currant extracts in milk product. J. Food Sci., 69(9), S351-356.

Stankiewicz, J. (2009). Jakość mlecznych napojów fermentowanych suplementowanych dodatkami pochodzenia roślinnego [The quality of fermented milk drinks supplemented with plant extracts]. Zesz. Nauk. Akad. Morsk. Gdyn., 61, 39-44 [in Polish].

Tamime, A. Y., Robinson, R. K. (1999). Yoghurt. Science and technology. Cambridge: Woodhead Publ.

Virtanen, T., Pihlanto, A., Akkanen, S., Korhonen, H. (2007). Development of antioxidant activity in milk whey during fermentation with lactic acid bacteria. J. Appl. Microbiol., 102(1), 106-115.

Zaręba, D., Ziarno, M. (2012). Dodatki: Dodatki modyfikujące smak i zapach [The additives: additives modifing taste and smell]. Forum Mlecz. Bizn., 14, 24 29 [in Polish].

Zhang, Y., Du, R., Wang, L., Zhang, H. (2010). The antioxidative effects of probiotic Lactobacillus casei Zhang on the hyperlipidemic rats. Eur. Food Res. Technol., 231(1), 151-158.

Zhang, S., Liu, L., Su, Y., Li, H., Sun, Q., Liang, X., Lv, J. (2011). Antioxidative activity of lactic acid bacteria in yogurt. Afr. J. Microbiol. Res., 5(29), 5194-5201.

Zulueta, A., Maurizi, A., Frígola, A., Esteve, M. J., Coli, R., Burini, G. (2009). Antioxidant capacity of cow milk, whey and deproteinized milk. Int. Dairy J., 19(6), 380-385. 


\section{OCENA AKTYWNOŚCI PRZECIWUTLENIAJĄCEJ MLECZNYCH NAPOJÓW FERMENTOWANYCH DOSTĘPNYCH W HANDLU}

\section{STRESZCZENIE}

Wstęp. Wolne rodniki są związane ze zwiększonym ryzykiem pewnych chorób, szczególnie nowotworów. Istnieją dowody naukowe, że dieta bogata w antyoksydanty może hamować negatywny wpływ wolnych rodników. Celem pracy była analiza właściwości przeciwutleniających wybranych naturalnych oraz smakowych mlecznych napojów fermentowanych dostępnych w handlu na polskim rynku, pochodzących od różnych producentów.

Materiał i metody. Analizie potencjału antyoksydacyjnego poddano następujące handlowe naturalne mleka fermentowane: 12 jogurtów, 12 kefirów, 2 maślanki, 2 zsiadłe mleka oraz napój turecki (ayran), a także następujące smakowe mleka fermentowane: 22 jogurty, 2 mleka acidofilne, 2 kefiry, maślankę oraz chłodnik warzywny. Ocenę potencjału antyoksydacyjnego przeprowadzono w dwóch powtórzeniach oraz dwukrotnie dla każdego produktu, poprzez określenie zdolności do redukcji jonów żelaza (FRAP) oraz zdolności wymiatania rodnika DPPH (wyrażonej jako ARP - aktywność przeciwrodnikowa).

Wyniki. Spośród wszystkich analizowanych naturalnych mlecznych napojów fermentowanych największą aktywnością przeciwutleniającą charakteryzowały się jogurty i kefiry. Obecność w produktach kultur probiotycznych Lactobacillus casei miała pozytywny wpływ na wyższy poziom zarówno wartości FRAP, jak i ARP. Właściwości przeciwutleniające smakowych mlecznych napojów fermentowanych były determinowane w głównej mierze rodzajem wprowadzonego dodatku smakowego oraz jego jakością (np. zawartością owoców). Najbardziej wartościowe pod względem tej cechy okazały się wsady: czekoladowy, kawowy, grejpfrutowy z dodatkiem ekstraktu zielonej herbaty, jak również: jagodowe, owoców leśnych, truskawkowe, z dodatkiem wiśni i czarnej porzeczki.

Wnioski. Głównymi czynnikami wpływającymi na właściwości antyoksydacyjne mlek fermentowanych okazały się: zawartość białka i mikroflory probiotycznej, a także rodzaj i jakość wsadów smakowych.

Słowa kluczowe: jogurt, kefir, maślanka, probiotyki, aktywność przeciwutleniająca

For citation - Do cytowania

Najgebauer-Lejko, D., Sady, M. (2015). Estimation of the antioxidant activity of the commercially available fermented milks. Acta Sci. Pol. Technol. Aliment., 14(4), 387-396. DOI: 10.17306/J.AFS.2015.4.38 